\title{
A novel therapeutic strategy using extracorporeal membrane oxygenation in patients with anti-neutrophil cytoplasmic antibodies-associated vasculitis: a case report and literature review
}

\author{
Li Wang ${ }^{1}$, Jiang Wang ${ }^{2}$, Yi Xu${ }^{2}$, Jie Jiao ${ }^{3}$, Lixin $\mathrm{Xie}^{2}$, Guoxin $\mathrm{Mo}^{2}$ \\ ${ }^{1}$ Department of Critical Care Medicine, The First Medical Center of PLA General Hospital, Haidian District, Beijing, China; ${ }^{2}$ Department of \\ Pulmonary \& Critical Care Medicine, The Eighth Medical Center of PLA General Hospital, Haidian District, Beijing, China; ${ }^{3}$ Department of \\ Critical Care Medicine, Hainan Hospital of PLA General Hospital, Sanya, China \\ Correspondence to: Guoxin Mo, MD, PhD. Department of Pulmonary \& Critical Care Medicine, The Eighth Medical Center of PLA General \\ Hospital, Haidian District, Beijing 100091, China. Email: 13661005996@139.com.
}

\begin{abstract}
Diffuse alveolar hemorrhage (DAH) secondary to anti-neutrophil cytoplasmic antibodies (ANCA)-associated vasculitis (AAV) often results in severe respiratory failure which requires emergent management. In patients who are resistant to traditional mechanical respiratory support, extracorporeal membrane oxygenation (ECMO) can be used to maintain gas exchange, thereby providing time for the administration of immunosuppressive therapy to control the inflammation. Herein, we report the application of ECMO to support an adult patient with AAV complicated by severe respiratory failure due to DAH. Similar cases in the literature were identified and discussed. The patient in our case study was successfully treated with ECMO in the acute phase and relieved by immunosuppressive therapy after withdrawal of ECMO. A search in the PubMed database revealed 32 similar cases with DAH, of which 11 cases were microscopic polyangiitis (MPA), 2 cases were eosinophilic granulomatosis with polyangiitis (EGPA), and 19 cases were granulomatosis with polyangiitis (GPA). These patients were all treated with ECMO. Therefore, to date, we identified 33 patients who were effectively treated with ECMO, including 13 (39.4\%) males and $20(60.6 \%)$ females, with a ratio of $1: 1.54$. The average age was $32.4 \pm 17.5$ and $36.0 \pm 16.1$ years for males and females, respectively $(\mathrm{t}=0.610, \mathrm{P}=0.547)$. Most patients received ECMO on the first day of admission to the intensive care unit (ICU) and it appeared that early initiation of ECMO was associated with a shorter duration of ECMO. In general, complications of ECMO in these patients were mild and were not often seen in the clinical setting. This study suggested that early recognition of respiratory failure and referral for ECMO are vital to achieve a satisfactory outcome in AAV patients with DAH.
\end{abstract}

Keywords: Anti-neutrophil cytoplasmic antibodies-associated vasculitis (ANCA-AAV); extracorporeal membrane oxygenation (ECMO); diffuse alveolar hemorrhage; respiratory failure; case report

Submitted May 24, 2021. Accepted for publication Jul 19, 2021.

doi: $10.21037 /$ atm-21-3133

View this article at: https://dx.doi.org/10.21037/atm-21-3133

(c) Annals of Translational Medicine. All rights reserved. 


\section{Introduction}

Anti-neutrophil cytoplasmic antibodies (ANCAs) are autoantibodies specific for antigens located in the cytoplasmic granules of neutrophils and the lysosomes of monocytes $(1,2)$. ANCA-associated vasculitis (AAV) is a group of systemic disorders characterized by inflammation and necrosis of small and medium size vessels, and is the major cause of vasculitis affecting the lungs. There are three types of AAV including microscopic polyangiitis (MPA), granulomatosis with polyangiitis (GPA), and eosinophilic granulomatosis with polyangiitis (EGPA). As the disease progresses, the disruption of the alveolar-capillary basement membrane accelerates the extravasation of red blood cells into the alveolar spaces (3), resulting in diffuse alveolar hemorrhage (DAH). DAH is the most serious complication of AAV and is one of the strongest predictors of early mortality $(4,5)$. Extracorporeal membrane oxygenation (ECMO) is an efficient method to correct hypoxemia in patients with severe acute respiratory failure, especially when conventional mechanical ventilation fails or when there is insufficient time to treat the underlying pathology $(6,7)$. Recent technological advances have allowed for the application of ECMO in AAV-related DAH, in addition to traditional immune-suppressors and corticosteroids, even if a certain degree of systemic anticoagulation is required. Therefore, the fine balance between bleeding and anticoagulation is crucial. Herein, we present a case report of a 56-year-old patient presenting with $\mathrm{AAV}$-related $\mathrm{DAH}$ who was successfully supported with ECMO. In addition, a review of the literature was conducted to further understand the utilization of ECMO in DAH. We present the following article in accordance with the CARE reporting checklist (available at https://dx.doi.org/10.21037/atm-21-3133).

\section{Case presentation}

A 56-year-old Chinese man presenting with cough, expectoration and chest tightness was admitted to the Department of Pulmonology at The First Medical Center of PLA General Hospital on Sep 29, 2017. Two months earlier, the patient experienced cough and expectoration, accompanied by left chest pain and chest tightness. The chest computed tomography (CT) scan showed bilateral lower lung field interstitial fibrosis (Figure 1A,1B). While the patient had been evaluated and treated empirically by antibiotics several times outside our department, the dyspnea persisted and was gradually aggravated with dizziness. Past medical history included hypertension with regular treatment. All procedures performed in studies involving human participants were in accordance with the ethical standards of the institutional and/or national research committee(s) and with the Helsinki Declaration (as revised in 2013). This study was approved by the local Ethics Committee of the Chinese PLA General Hospital (Beijing, China) (No. S2020-141-01). The patient provided written informed consent for the publication of any associated data and accompanying images.

On physical examination, the patient's temperature was $38.7^{\circ} \mathrm{C}$, pulse rate was 106 beats per minute, respiratory rate was 46 breaths per minute, and blood pressure was $135 / 79 \mathrm{mmHg}$. The breath sounds of double lungs had coarse crackles, a small number of wet rales could be auscultated in the left lower lung and no dry rales and pleural frictions could be heard. The cardiovascular, abdominal, musculoskeletal, and neurologic examinations were normal. The chest radiograph (Figure 1C) revealed multiple patchy lesions and diffuse alveolar infiltration. Laboratory data on admission included the following: a hemoglobin count of $87 \mathrm{~g} / \mathrm{L}$, a white blood cell (WBC) count of $16.77 \times 10^{9}$ cells/L, and a platelet count of $389 \times 10^{9}$ cells $/ \mathrm{L}$. Analysis of infectious indicators revealed $10.61 \mathrm{mg} / \mathrm{d} L$ C-reactive protein, $0.717 \mathrm{ng} / \mathrm{mL}$ procalcitonin, and $118.10 \mathrm{pg} / \mathrm{mL}$ interleukin-6. The biochemical indicators showed creatinine levels at $325 \mathrm{mmol} / \mathrm{L}$, blood urea nitrogen (BUN) levels at $12.5 \mathrm{mmol} / \mathrm{L}$, lactate dehydrogenase levels at 307 units/L, and plasma $\mathrm{N}$-terminal pro-B-type natriuretic peptide (BNP) levels at $1,046 \mathrm{pg} / \mathrm{mL}$. Initial arterial blood gas analysis showed that $\mathrm{pH}$ was 7.45 , partial pressure of carbon dioxide $\left(\mathrm{PaCO}_{2}\right)$ was $28 \mathrm{mmHg}$, partial pressure of oxygen $\left(\mathrm{PaO}_{2}\right)$ was $42 \mathrm{mmHg}$, bicarbonate $\left(\mathrm{HCO}_{3-}\right)$ was $19.5 \mathrm{mmol} / \mathrm{L}$, and oxygen $\left(\mathrm{O}_{2}\right)$ saturation was $80 \%$, suggesting acute respiratory failure.

On the first day of admission, the patient was administered oxygen via a face mask and the pulse oxygen saturation was maintained $80 \%$. To improve hypoxemia, non-invasive positive pressure ventilation [mode: spontaneous/timed ( $/ \mathrm{T})$; inspiratory positive airway pressure (IPAP) $7 \mathrm{cmH}_{2} \mathrm{O}$; expiratory positive airway pressure (EPAP) $4 \mathrm{cmH}_{2} \mathrm{O}$; respiratory rate 18 breaths/ minute; fraction of inspired oxygen $\left(\mathrm{FiO}_{2}\right)$ 80\%] and continuous invasive mechanical ventilation [mode: volumecontrolled ventilation; tidal volume $450 \mathrm{~mL}$; positive end expiratory pressure (PEEP) $6 \mathrm{cmH}_{2} \mathrm{O}$; respiratory rate 18 breaths/minute; $\mathrm{FiO}_{2}$ 100\%] were successively applied. 

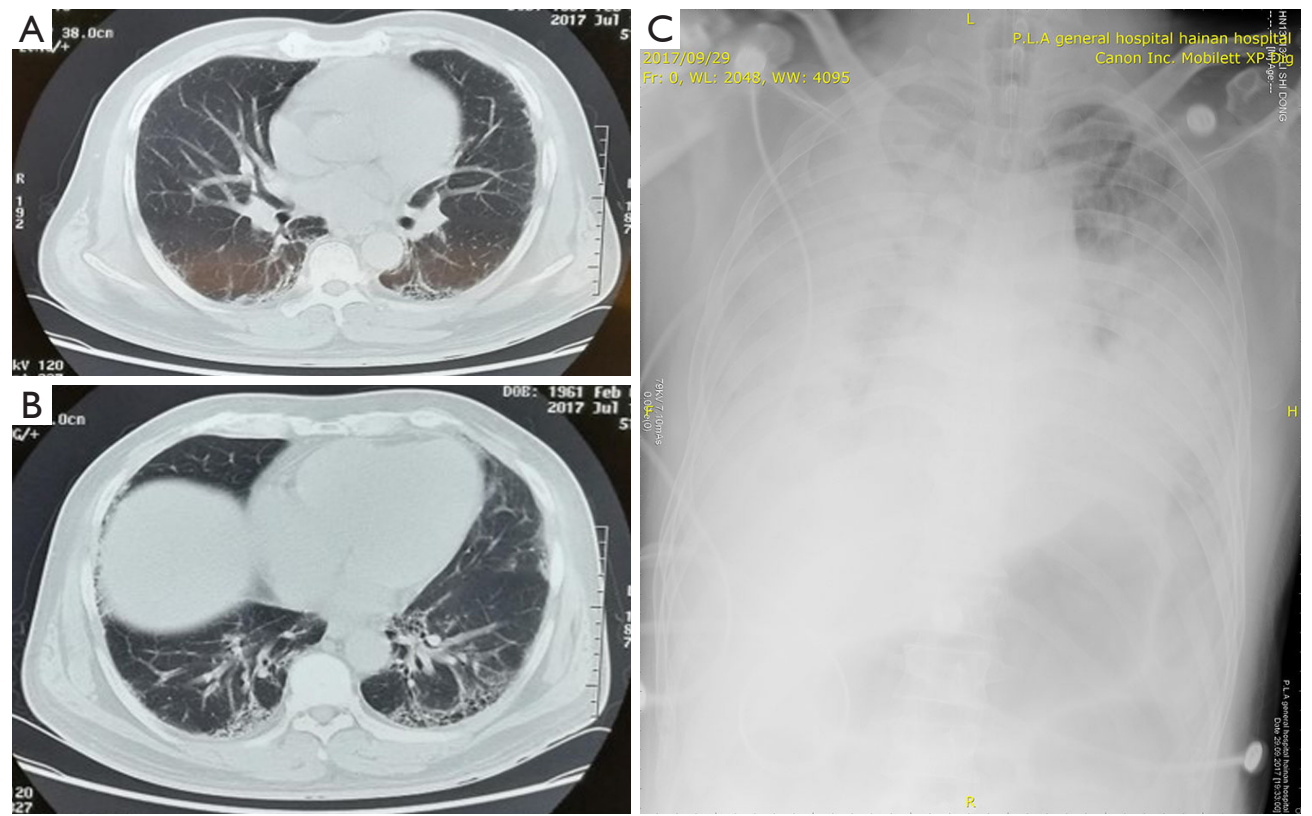

Figure 1 Chest image of a case of AAV. (A,B) CT scan of the chest showing interstitial fibrosis in both bilateral lower lung fields. (C) Chest radiograph showing bilateral diffuse alveolar opacities. AAV, ANCA-associated vasculitis; ANCA, anti-neutrophil cytoplasmic antibodies; CT, computed tomography.
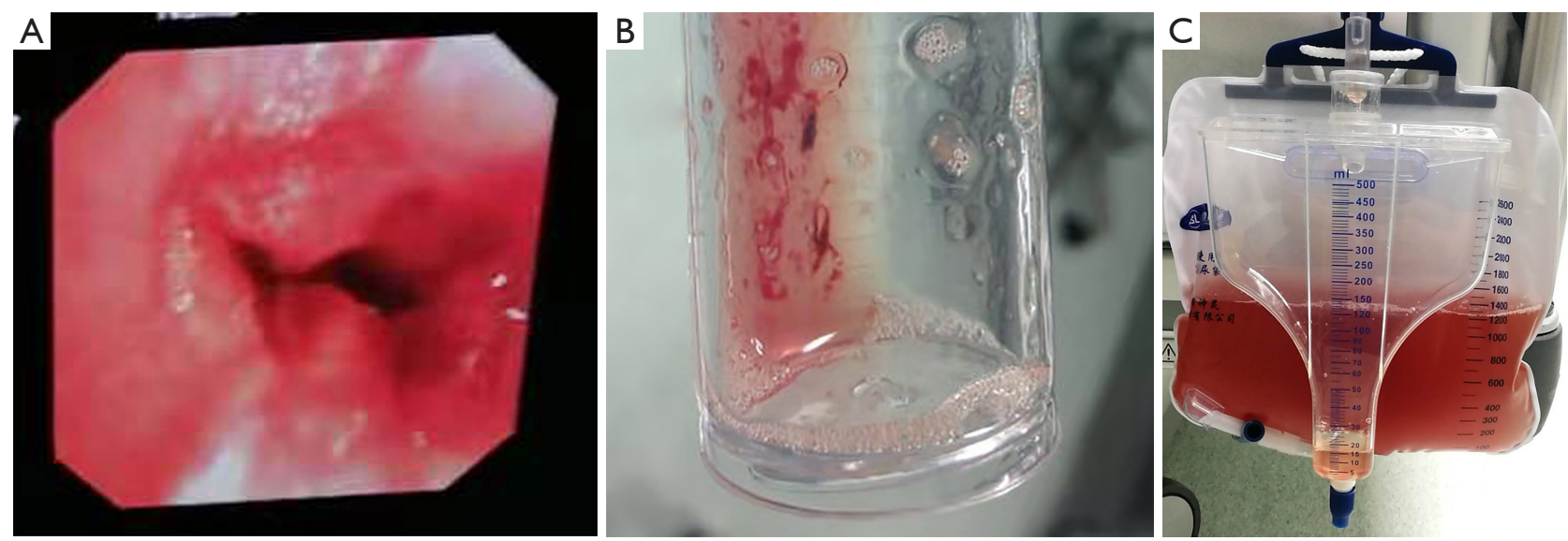

Figure 2 Bronchial mucosal hyperemia. (A) View through the bronchoscope shows intratracheal bleeding. (B) Stained bloody secretions. (C) Gross hematuria was observed.

The preliminary diagnosis was pulmonary infection with pulmonary interstitial fibrosis, pending examination of rheumatic immune-related antibodies. Treatment with methylprednisolone $80 \mathrm{mg}$ once daily, moxifloxacin, ganciclovir, meropenem, caspofungin, linezolid, and gamma globulin were commenced immediately to control infection, suppress inflammation, and regulate immunology. Meanwhile, continuous renal replacement therapy (CRRT) was administered to remove the inflammatory mediators and reduced oxygen consumption in the organs. Bronchoscopy was performed to detect airway hyperemia (Figure $2 A$ ) and to remove the stained bloody secretions (Figure 2B). Gross hematuria was visible (Figure 2C). Despite the continuous mechanical ventilation on an $\mathrm{FiO}_{2}$ of $100 \%$, hypoxemia failed to improve and oxygen saturation and the $\mathrm{PaO}_{2} / \mathrm{FiO}_{2}$ ratio continued to deteriorate. 
Table 1 The treatment regimen during the patient's hospital stay

\begin{tabular}{lccc}
\hline Events & Time & Hospital day & Dose \\
\hline ECMO & & $4-11$ & \\
Ventilation & & $1-17$ & \\
CRRT & 4 & $4,5,6,7$ & \\
MePr & Once per day & $1-10$ & $80 \mathrm{mg}$ \\
& Once per day & 11 & $200 \mathrm{mg}$ \\
& Once per day & $12-13$ & $500 \mathrm{mg}$ \\
& Once per day & $14-20$ & $80 \mathrm{mg}$ \\
& Once per day & $21-22$ & $60 \mathrm{mg}$ \\
& Once per day & $23-$ discharge & $40 \mathrm{mg}$ (oral) \\
& Once per day & $3-6$ & $20 \mathrm{~g}$ \\
& Once per day & $13-15$ & $10 \mathrm{~g}$ \\
\hline
\end{tabular}

ECMO, extracorporeal membrane oxygenation; CRRT, continuous renal replacement therapy; MePr, methylprednisolone; GG, gamma globulin.

The patient was transferred to the intensive care unit (ICU) on the day of admission, and on hospital day 4, the decision was made to initiate emergency veno-venous ECMO (V-V ECMO) for respiratory support. The ECMO unit was established at the beside of the ICU, and consisted of a centrifugal pump, Oxygenator (Sorin Group, Italy), and tubes. A $21 \mathrm{~F}$ cannula was penetrated into the patient's right femoral vein and a $17-\mathrm{Fr}$ cannula was placed in the patient's right internal jugular vein. The optimal location of the cannulas was confirmed by transthoracic echocardiography (TTE) to minimize recirculation. The blood was then circulated from the femoral vein and returned through the internal jugular vein. At beginning of ECMO, the average flow rate was set at $3.5 \mathrm{~L} / \mathrm{min}$, the sweep gas flow rate was set at $8 \mathrm{~L} / \mathrm{min}$, and the rotation rate was set at 2,500 rpm. Despite the risk of re-bleeding, a maintenance dose of heparin was applied until termination of ECMO. There was no further bleeding from the lungs, and the low dosage unfractionated heparin kept the activated clotting time (ACT) at about 200 seconds and the activated partial thromboplastin time (APTT) at about 50 seconds. During his support of extracorporeal circulation, flows were maintained between 3.5 and $3.8 \mathrm{~L} / \mathrm{min}$, and the gas at about $8 \mathrm{~L} / \mathrm{min}$. The ventilator settings were adjusted as follows: mode was pressure control ventilation positive, end expiratory pressure $12 \mathrm{cmH}_{2} \mathrm{O}$, respiratory rate 10 breaths per minute, and $\mathrm{FiO}_{2}$ at $30 \%$. Due to the changes in pharmacokinetics, the dosage and time of drug administration were adjusted, with the dose of methylprednisolone increased to $120 \mathrm{mg}$ daily from hospital day 4 to day 12 .

The patient remained stable on hospital day 11 (ECMO day 8), and was able to remain without ECMO for 6 hours with an oxygen saturation in the mid-95\% range and PEEP at $8 \mathrm{cmH}_{2} \mathrm{O}$. The ECMO unit was removed successfully and mechanical ventilation support was continued. The patient's bleeding had stopped, the oxygenation had improved, and chest imaging also shown improvements. On the $17^{\text {th }}$ hospital day, the tracheal intubation was removed.

On hospital day 9 of intubation, the laboratory data showed that the patient was positive for cytoplasmic ANCA antibodies (C-ANCA), perinuclear ANCA antibodies (P-ANCA), antimyeloperoxidase antibodies (anti-MPO), and anti-cardiolipin antibodies. However, he was negative for anti-glomerular basement membrane antibodies (anti-GBM). Combined with the previous results, a diagnosis of AAV was confirmed (MPA). Thus, the dose of methylprednisolone was increased to $500 \mathrm{mg}$ daily from hospital day 13 to day 14 and decreased to $80 \mathrm{mg}$ daily from hospital days 15 to 21 . When the methylprednisolone was decreased to $40 \mathrm{mg}$, an equal dose was administered orally until discharge. Blood, sputum culture, excrement, and urine were tested daily to assess for infections and guide the use of antibiotics. The major events of therapy during the hospital stay are summarized in Table 1. The patient gradually improved and the chest radiographs also gradually showed improvements (Figure 3). Thus, he was discharged to the Department of Rheumatology and where his treatment continued. The patient has since recovered well.

\section{Literature review}

A detailed search in the PubMed database revealed 32 similar cases with DAH, of which 11 were MPA, 2 were EGPA, and 19 were GPA. These patients were all treated with ECMO (see Table 2 for details). Thus, in total, we reported 33 patients who were successfully treated with ECMO. Of these patients, there were 13 (39.4\%) males and $20(60.6 \%)$ females, with a ratio of $1: 1.54$. The average age was $32.4 \pm 17.5$ and $36.0 \pm 16.1$ years for males and females, respectively $(\mathrm{t}=0.610, \mathrm{P}=0.547)$.

\section{Discussion}

ANCA are a family of autoantibodies which can cause a potentially life-threatening systemic necrotizing small vessel 


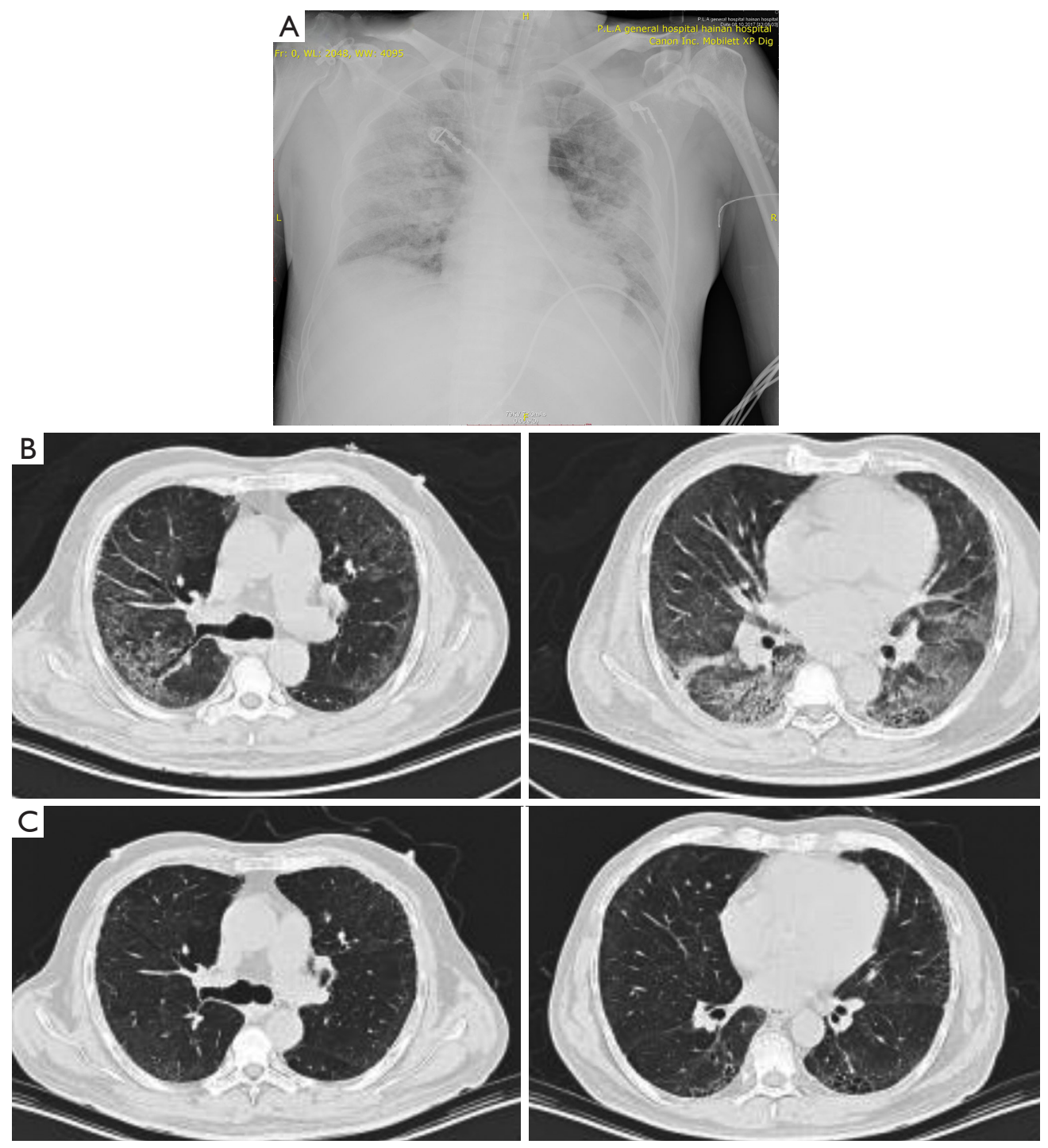

Figure 3 Chest radiography monitoring of a patient with AAV. (A) A chest radiograph taken at the conclusion of ECMO therapy. (B) CT scan of the chest after 19 days in hospital, and (C) after 1 month. AAV, ANCA-associated vasculitis; ANCA, anti-neutrophil cytoplasmic antibodies; ECMO, extracorporeal membrane oxygenation; CT, computed tomography.

vasculitis referred to as AAV. This group of autoimmune diseases involve both small and medium vessels, and includes GPA, MPA, and EGPA (1). When AAV affects other organs, diagnosis and treatment tend to be delayed due to its nonspecific clinical manifestations. In a systematic review, the authors enrolled 56 studies on 1,422 AAV patients treated with Rituximab (RTX). Complete or partial remission rates over $80 \%$ were reported in most studies. The total relapse rate was $30 \%$. Main adverse events included infections and infusion reactions (35).

$\mathrm{DAH}$ is one of the most serious complications of AAV.
The main clinical manifestations are hemoptysis, or unexplained dyspnea, and an abrupt drop in hemoglobin levels. Although hemoptysis is a characteristic symptom, it is not observed in about one-third of cases $(36,37)$. The major diagnostic criteria include assessment of ANCArelated antibodies and bronchoalveolar lavage fluid, radiology examinations, and lung biopsies (38). Despite surgical lung biopsies representing the gold standard (39), it is not commonly recommended. The common causes of DAH are related to ANCA-related vasculitis, drugs (especially anti-thyroid drugs such as propylthiouracil), 
Table 2 A summary of 33 patients with ANCA-associated vasculitis treated by EMCO

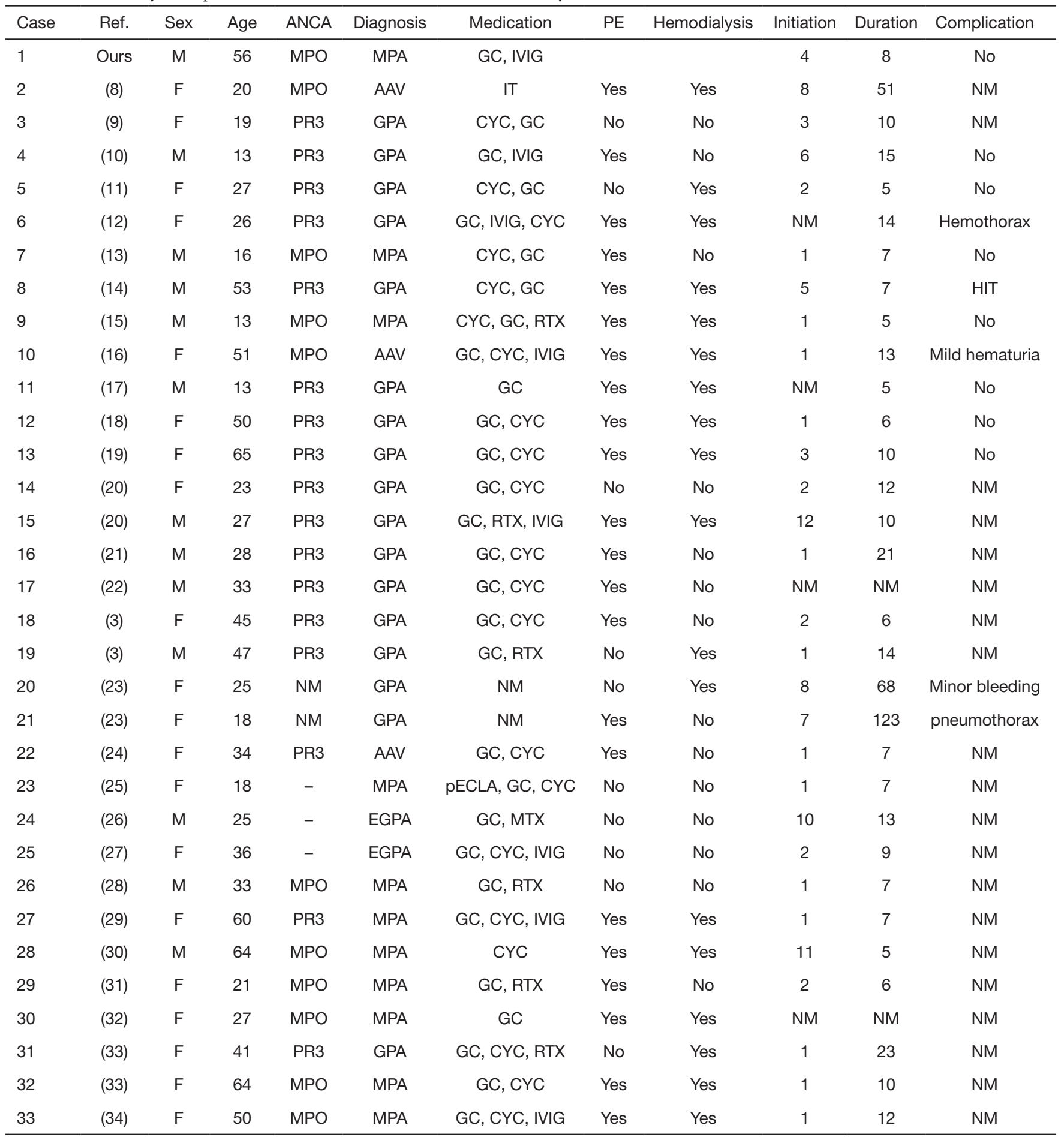

Initiation refers to the day from transferred into ICU to ECMO; Duration refers to time on ECMO therapy (days). ANCA, anti-neutrophil cytoplasmic antibodies; ECMO, extracorporeal membrane oxygenation; M, male; F, female; ANCA, anti-neutrophil cytoplasmic antibodies; MPO, anti-myeloperoxidase antibodies; PR3, proteinase 3; NM, not mentioned; MPA, microscopic polyangiitis; AAV, ANCA-associated vasculitis; GPA, granulomatosis with polyangiitis; EGPA, eosinophilic granulomatosis with polyangiitis; GC, glucocorticoids; IVIG, intravenous immunoglobulin; IT immunosuppressive therapy; CYC, cyclophosphamide; RTX, rituximab; pECLA, pumpless extracorporeal lung assist; HIT, heparin-induced thrombocytopenia; PE, plasma exchange; -, represents negative. 
infections, connective tissue diseases such as systemic lupus erythematosus (SLE), or other small vasculitis (40). Once DAH is diagnosed, it requires pulse corticosteroids, intravenous cyclophosphamide, and rituximab. Moreover, plasma exchanges can be used in patients who suffer from anti-GBM disease, AAV, SLE, and occasionally in patients with other autoimmune disorders $(41,42)$. In some cases, DAH complications can affect vital organs, and in such cases, invasive mechanical ventilation, CRRT, and ECMO may be used as temporary adjunctive therapy (12).

ECMO is frequently used in the management of critical diseases, especially cardiogenic shock, cardiac arrest, fulminant myocarditis, and rejection of heart transplantation therapy $(43,44)$. ECMO manages the task of gas exchange so that the lungs can remain in a resting state, thereby facilitating the patient's rehabilitation. For hypoxemia caused by DAH, mechanical ventilation is usually the first-line treatment of choice in clinics. For more severe refractory cases, ECMO may be used as a short-term supporting method (12).

Herein, we described a patient with AAV-related DAH who suffered from severe respiratory failure. This patient was successfully supported by ECMO which was an essential part of his treatment and evidently improved his prognosis. Mechanical ventilation support is provided to patients who experience persistent hypoxia and respiratory failure. When conventional mechanical ventilation fails, ECMO is used to provide oxygenation immediately (indications for use: oxygenation index less than $80 \%$, ventilation failure in the prone position, life-threatening pneumothorax or hemoptysis). After 2 days of support with V-V ECMO, the patient's pulse oxygen increased and was maintained above $95 \%$. The alveolar hemorrhage did not deteriorate and was resolved after ECMO therapy. These results demonstrated that while the application of ECMO is not suitable for etiological treatment, it confers life-supporting benefits which allows crucial time for immunosuppressive agents to take effect and terminate the pathophysiological processes of the underlying disease. Therefore, ECMO may be the essential strategy to sustain life in patients with acute respiratory failure if conventional therapy fails or if there is insufficient time to treat the primary disease. Different from the case in a report by Goel et al. (24), ours progressed relatively slow, which perhaps help us to save his life.

The standard treatment of AAV-related DAH comprises of high doses of glucocorticoids in combination with cyclophosphamide. The recommended dosage of methylprednisolone ranges from 0.5 to $1.0 \mathrm{~g}$ per day for $1-3$ days or $15 \mathrm{mg} / \mathrm{kg}$ (maximum dose per pulse of
$1 \mathrm{~g})$ per dose for 1-3 days (45). In our case, the dose of methylprednisolone (80-120 $\mathrm{mg}$ per day) during ECMO was less than the recommended dose. However, the patient's condition improved gradually, suggesting that the application of ECMO can decrease the dose of glucocorticoids required and it may have a direct beneficial effect in improving the outcomes of DAH. The optimization of glucocorticoid therapy with ECMO warrants further investigation.

In addition, due to the rarity and the high mortality rate of DAH secondary to $\mathrm{AAV}$, there have been few cases detailing the use of ECMO in this condition. The comprehensive clinical data of fifteen cases from the literature are listed in Table 2.

Although we were successful in treating this patient, there are a few noteworthy points. First, AAV-related DAH may be misdiagnosed as pulmonary interstitial fibrosis according to chest imaging. Second, the patient's pulmonary hemorrhage with renal function was easily misdiagnosed as Good-pasture syndrome. Third, when combined with organ dysfunction such as acute respiratory distress syndrome (ARDS), it is necessary to support the vital organs in a timely manner, especially with the application of ECMO which can allow for valuable time for the diagnosis and treatment of the primary disease.

This report also summarized 33 patients with AAV who were treated with ECMO. Most of these patients received ECMO on the first day of admission to the ICU and there appeared be a trend that early initiation of ECMO was associated with a shorter duration of ECMO therapy. In general, complications of ECMO in these patients were mild and not often seen in practice. In a retrospective analysis, Seeliger et al. reported 12 patients with GPA and 3 patients with MPA who received ECMO therapy. However, the clinical details of each patient were not provided (46). In addition, there were other similar case reports which did not provide full text articles and thus, could not be incorporated into our literature review $(47,48)$. Nonetheless, to the best of our knowledge, there have been least 48 cases of AAV which have been successfully treated with ECMO therapy. These 48 cases provide valid support for similar patients to receive ECMO as early as possible.

Our patient expressed his satisfactory for our service and hoped to do follow-up regularly.

\section{Conclusions}

The emergent condition caused by AAV-related DAH 
can be effectively managed by ECMO. In our case study, supporting respiratory function with ECMO effectively improved the potentially fatal hypoxemia caused by pulmonary hemorrhage. With the improvement of oxygenation, effective control of underlying disease allowed for the removal of extracorporeal circulation and extubation. In the past case reports, no life-threatening hemorrhage events occurred during ECMO intervention. Although ECMO is a highly invasive procedure with a significant risk of bleeding complications, early initiation of ECMO and meticulous care during treatment may be a novel and lifesaving strategy in patients with AAV-related DAH who fail to respond to conventional mechanical ventilation.

\section{Acknowledgments}

Funding: None.

\section{Footnote}

Reporting Checklist: The authors have completed the CARE reporting checklist. Available at https://dx.doi. org/10.21037/atm-21-3133

Conflicts of Interest: All authors have completed the ICMJE uniform disclosure form (available at https://dx.doi. org/10.21037/atm-21-3133). The authors have no conflicts of interest to declare.

Ethical Statement: The authors are accountable for all aspects of the work in ensuring that questions related to the accuracy or integrity of any part of the work are appropriately investigated and resolved. This study was approved by the local Ethics Committee of the Chinese PLA General Hospital (Beijing, China) (No. S2020-14101). The patient provided written informed consent for the publication of any associated data and accompanying images. All procedures performed in studies involving human participants were in accordance with the ethical standards of the institutional and/or national research committee(s) and with the Helsinki Declaration (as revised in 2013).

Open Access Statement: This is an Open Access article distributed in accordance with the Creative Commons Attribution-NonCommercial-NoDerivs 4.0 International License (CC BY-NC-ND 4.0), which permits the noncommercial replication and distribution of the article with the strict proviso that no changes or edits are made and the original work is properly cited (including links to both the formal publication through the relevant DOI and the license). See: https://creativecommons.org/licenses/by-nc-nd/4.0/.

\section{References}

1. Jennette JC, Falk RJ, Bacon PA, et al. 2012 revised International Chapel Hill Consensus Conference Nomenclature of Vasculitides. Arthritis Rheum 2013;65:1-11.

2. Alba MA, Jennette JC, Falk RJ. Pathogenesis of ANCAAssociated Pulmonary Vasculitis. Semin Respir Crit Care Med 2018;39:413-24.

3. Delvino P, Monti S, Balduzzi S, et al. The role of extracorporeal membrane oxygenation (ECMO) in the treatment of diffuse alveolar haemorrhage secondary to ANCA-associated vasculitis: report of two cases and review of the literature. Rheumatol Int 2019;39:367-75.

4. Hogan SL, Nachman PH, Wilkman AS, et al. Prognostic markers in patients with antineutrophil cytoplasmic autoantibody-associated microscopic polyangiitis and glomerulonephritis. J Am Soc Nephrol 1996;7:23-32.

5. Haworth SJ, Savage CO, Carr D, et al. Pulmonary haemorrhage complicating Wegener's granulomatosis and microscopic polyarteritis. Br Med J (Clin Res Ed) $1985 ; 290: 1775-8$.

6. Lewandowski K. Extracorporeal membrane oxygenation for severe acute respiratory failure. Crit Care 2000;4:156-68.

7. Tulman DB, Stawicki SPA, Whitson BA, et al. Venovenous ECMO: a synopsis of nine key potential challenges, considerations, and controversies. BMC Anesthesiol 2014;14:65.

8. Hartmann A, Nordal KP, Svennevig J, et al. Successful use of artificial lung (ECMO) and kidney in the treatment of a 20-year-old female with Wegener's syndrome. Nephrol Dial Transplant 1994;9:316-9.

9. Loscar M, Hummel T, Haller M, et al. ARDS and Wegener granulomatosis. Anaesthesist 1997;46:969-73.

10. Hernandez ME, Lovrekovic G, Schears G, et al. Acute onset of Wegener's granulomatosis and diffuse alveolar hemorrhage treated successfully by extracorporeal membrane oxygenation. Pediatr Crit Care Med 2002;3:63-6.

11. Rosengarten A, Elmore P, Epstein J. Long distance road transport of a patient with Wegener's Granulomatosis and respiratory failure using extracorporeal membrane oxygenation. Emerg Med (Fremantle) 2002;14:181-7. 
12. Ahmed SH, Aziz T, Cochran J, et al. Use of extracorporeal membrane oxygenation in a patient with diffuse alveolar hemorrhage. Chest 2004;126:305-9.

13. Agarwal HS, Taylor MB, Grzeszczak MJ, et al. Extra corporeal membrane oxygenation and plasmapheresis for pulmonary hemorrhage in microscopic polyangiitis. Pediatr Nephrol 2005;20:526-8.

14. Balasubramanian SK, Tiruvoipati R, Chatterjee S, et al. Extracorporeal membrane oxygenation with lepirudin anticoagulation for Wegener's granulomatosis with heparin-induced thrombocytopenia. ASAIO J 2005;51:477-9.

15. Di Maria MV, Hollister R, Kaufman J. Case report: severe microscopic polyangiitis successfully treated with extracorporeal membrane oxygenation and immunosuppression in a pediatric patient. Curr Opin Pediatr 2008;20:740-2.

16. Guo Z, Li X, Jiang LY, et al. Extracorporeal membrane oxygenation for the management of respiratory failure caused by diffuse alveolar hemorrhage. J Extra Corpor Technol 2009;41:37-40.

17. Joseph M, Charles AG. Early extracorporeal life support as rescue for Wegener granulomatosis with diffuse alveolar hemorrhage and acute respiratory distress syndrome: a case report and literature review. Pediatr Emerg Care 2011;27:1163-6.

18. Barnes SL, Naughton M, Douglass J, et al. Extracorporeal membrane oxygenation with plasma exchange in a patient with alveolar haemorrhage secondary to Wegener's granulomatosis. Intern Med J 2012;42:341-2.

19. Hohenforst-Schmidt W, Petermann A, Visouli A, et al. Successful application of extracorporeal membrane oxygenation due to pulmonary hemorrhage secondary to granulomatosis with polyangiitis. Drug Des Devel Ther 2013;7:627-33.

20. Yusuff H, Malagon I, Robson K, et al. Extracorporeal membrane oxygenation for Life-threatening ANCApositive pulmonary capillaritis. A review of UK experience. Heart Lung Vessel 2015;7:159-67.

21. Rawal G, Kumar R, Yadav S. ECMO Rescue Therapy in Diffuse Alveolar Haemorrhage: A Case Report with Review of Literature. J Clin Diagn Res 2016;10:OD10-1.

22. Vanoli J, Riva M, Vergnano B, et al. Granulomatosis with polyangiitis presenting with diffuse alveolar hemorrhage requiring extracorporeal membrane oxygenation with rapid multiorgan relapse: A case report. Medicine (Baltimore) 2017;96:e6024.

23. Yin K, March RJ, Hoopes CW, et al. Extracorporeal membrane oxygenation in the management of granulomatosis with polyangiitis. J Card Surg 2021;36:743-47.

24. Goel MK, Chauhan M, Kumar A, et al. A Case of Refractory Hypoxemic Respiratory Failure due to Antineutrophil Cytoplasmic Antibodies-associated Diffuse Alveolar Hemorrhage Rescued by Extracorporeal Membrane Oxygenation. Indian J Crit Care Med 2020;24:879-81.

25. Renner A, Neukam K, Rösner T, et al. Pumpless extracorporeal lung assist as supportive therapy in a patient with diffuse alveolar hemorrhage. Int J Artif Organs 2008;31:279-81.

26. Ammirati E, Cipriani M, Musca F, et al. A life-threatening presentation of eosinophilic granulomatosis with polyangiitis. J Cardiovasc Med (Hagerstown) 2016;17 Suppl 2:e109-11.

27. Cui N, Su L, Wang H, et al. A case report of ChurgStrauss syndrome presenting with cardiogenic shock treated with extracorporeal membrane oxygenation. Medicine (Baltimore) 2015;94:e1757.

28. Kundu S, Sharma S, Minhas R, et al. Acute Respiratory Distress Syndrome Requiring Extracorporeal Membrane Oxygenation as the Initial Presentation of Antineutrophillic Cytoplasmic Auto-antibody Positive Vasculitis. Cureus 2019;11:e6135.

29. Tong CK, Jin J, Du Q. Use of Thromboelastogram in Venovenous Extracorporeal Membrane Oxygenation for a Patient with Pulmonary Hemorrhage due to Microscopic Polyangiitis. Case Rep Crit Care 2019;2019:7241264.

30. Lee CF, Huang CT, Ruan SY. Endotracheal tube clamping and extracorporeal membrane oxygenation to resuscitate massive pulmonary haemorrhage. Respirol Case Rep 2018;6:e00321.

31. Kelly D, Makkuni D, Ail D. Rare cause of respiratory failure in a young woman: isolated diffuse alveolar haemorrhage requiring extracorporeal membrane oxygenation. BMJ Case Rep 2017;2017:bcr2017219235.

32. Ortiz-Diaz EO. A 27-year-old woman presenting with refractory hypoxaemic respiratory failure, haemoptysis and thyrotoxicosis: a rare manifestation of propylthiouracil therapy. BMJ Case Rep 2014;2014:bcr2014204915.

33. Arnold S, Deja M, Nitschke M, et al. Extracorporeal membrane oxygenation in ANCA-associated vasculitis. Autoimmun Rev 2021;20:102702.

34. Zhong H. Chen JH, Li SQ, et al. extracorporeal membrane oxygenation for pulmonary hemorrhage in microscopic polyangiitis. Chin Med J (Engl) 2008;121:2622-3. 
35. Ayan G, Esatoglu SN, Hatemi G, et al. Rituximab for antineutrophil cytoplasmic antibodies-associated vasculitis: experience of a single center and systematic review of nonrandomized studies. Rheumatol Int 2018;38:607-622.

36. de Prost N, Parrot A, Picard C, et al. Diffuse alveolar haemorrhage: factors associated with in-hospital and longterm mortality. Eur Respir J 2010;35:1303-11.

37. Quadrelli S, Diana D, Solis M, et al. Immune diffuse alveolar hemorrhage: Clinical presentation and outcome. Respir Med 2017;129:59.

38. De Lassence A, Fleury-Feith J, Escudier E, et al. Alveolar hemorrhage. Diagnostic criteria and results in 194 immunocompromised hosts. Am J Respir Crit Care Med 1995;151:157-63.

39. King TE Jr. A lung biopsy is necessary in the management of ANCA-positive patients with chest roentgenographic abnormalities. Sarcoidosis Vasc Diffuse Lung Dis 1996;13:238-40.

40. Nasser M, Cottin V. Alveolar Hemorrhage in Vasculitis (Primary and Secondary). Semin Respir Crit Care Med 2018;39:482-93.

41. Klemmer PJ, Chalermskulrat W, Reif MS, et al. Plasmapheresis therapy for diffuse alveolar hemorrhage in patients with small-vessel vasculitis. Am J Kidney Dis 2003;42:1149-53.

42. Ravindran V, Watts RA. Pulmonary haemorrhage in ANCA-associated vasculitis. Rheumatology

Cite this article as: Wang L, Wang J, Xu Y, Jiao J, Xie L, Mo G. A novel therapeutic strategy using extracorporeal membrane oxygenation in patients with anti-neutrophil cytoplasmic antibodies-associated vasculitis: a case report and literature review. Ann Transl Med 2021;9(15):1267. doi: 10.21037/atm21-3133
2010;49:1410-2.

43. Okada N, Murayama H, Hasegawa H, et al. Peripheral Veno-Arterial Extracorporeal Membrane Oxygenation as a Bridge to Decision for Pediatric Fulminant Myocarditis. Artif Organs 2016;40:793-8.

44. Kittleson MM, Patel JK, Moriguchi JD, et al. Heart transplant recipients supported with extracorporeal membrane oxygenation: Outcomes from a single-center experience. J Heart Lung Transplant 2011;30:1250-6.

45. Souza AWS, Calich AL, Mariz HA, et al. Recommendations of the Brazilian Society of Rheumatology for the induction therapy of ANCAassociated vasculitis. Rev Bras Reumatol Engl Ed 2017;57 Suppl 2:484-96.

46. Seeliger B, Stahl K, Schenk H, et al. Extracorporeal Membrane Oxygenation for Severe ARDS Due to Immune Diffuse Alveolar Hemorrhage: A Retrospective Observational Study. Chest 2020;157:744-7.

47. Matsumoto T, Ueki K, Tamura S et al. Extracorporeal membrane oxygenation for the management of respiratory failure due to ANCA-associated vasculitis. Scand J Rheumatol 2000;29:195-7.

48. Mikami M, Mori Y, Yamazaki A, et al. Nihon Naika Gakkai Zasshi 2000;89:1651-3.

(English Language Editor: J. Teoh) 